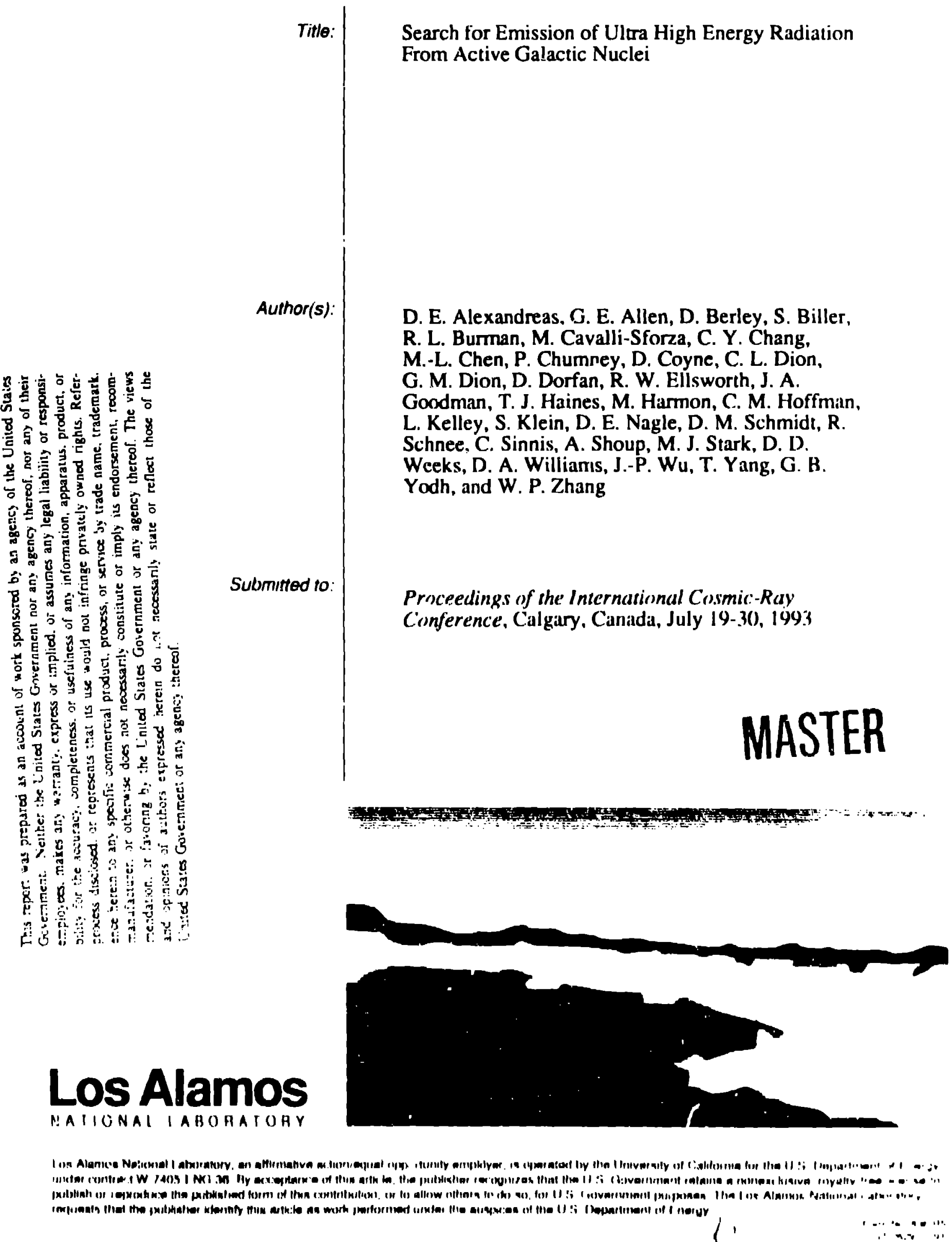




\title{
Search for Emission of Ultra High Energy Radiation from Active Galactic Nuclei
}

The CYGNUS Collaboration

D.E. Alexandreas, ${ }^{1,7}$ G.E. Allen, ${ }^{2}$ D. Berley, ${ }^{2,4}$ S. Biller, ${ }^{1}$ R. L. Burman, ${ }^{3}$ M. Cavalli-Sforsa, ${ }^{5}$ C.Y. Chang, ${ }^{2}$ M.-L. Chen, ${ }^{2}$ P. Chumney, ${ }^{1}$ D. Coyne, ${ }^{7}$ C. Dion, ${ }^{2}$ G.M. Dion, ${ }^{1,9}$ D. Dorfan, ${ }^{5}$ R.W. Ellsworth, J.A. Goodmar 2 T.J. Haines, M. Harmon, ${ }^{1}$ C.M. Hoffman, ${ }^{3}$ L. Kelley, ${ }^{5}$ S. Klein, ${ }^{5}$ D.E. Nagle, ${ }^{3}$ D.M. Schmidt, ${ }^{3}$ R. Schnee, ${ }^{5}$ C. Sinnis, ${ }^{3}$ A. Shoup, ${ }^{1}$ M.J. Stark, ${ }^{2}$ D.D. Weeks, ${ }^{3}$ D.A. Willians,; J.-P. WL:, ${ }^{6}$ T. Yang, ${ }^{5}$ G.B. Yodh, ${ }^{1}$ and W.P. Zhang ${ }^{3,10}$

1 The University of California, Irvine

2 The University of Maryland, College Park

${ }^{3}$ Los Alamos National Laboratory, Los Alamos, New Mezico

+ George Mason University, Fairfax, Virginia

s The University of California, Santa Cruz

${ }^{6}$ The University of California, Riverside

7 Now at Instituto Nazionale di Fisica Nucleare, Padova, Italy

* National Science Foundation, Washington, D.C.

'Now at ICRR, University of Tokyo, Tokyo, Japan

10 Now at NASA Goddard Space Flight Center, Greenbelt, MD

\begin{abstract}
A search for emission of ultra-high energy gamma radiation from 13 active galactic nuclei that were detected by EGRET, using the CYCNUS extensive airshower array, is described. The data et spans the period 1986 April 2 to 1993 January 1. The dats et has been searched for continuoun emisuion, emission on the tume acale of one week, and for emistion on the time scale of one day. No evidence for emisaion frum any of the $A C N$ on any of the time scales examined was found. The $90 \%$ C.L. upper limit to the continuous flux from Mrk 421 above $50 \mathrm{TeV}$ is $7.5 \times 10^{-1} \mathrm{~cm}^{-2},-1$
\end{abstract}

1. INTRODUCTION The Energetic Gamma Ray Experiment 'Telescope (BCRE'T) on the Compton Obaervatoty has observed high-energy gamma-ray emisaion from 18 active galactic nuerei (AGN) (Fichtel et al. 1083, Hartmnn et al. 1992). 'The gammn-iay spectra of these $A G N$ are quite harci, with differential spectral indices ranging from

1.7 to 2.4 (Fichtel et al. 1993). The high energy emission from some of these objerts has been obeerved to be variable, with meneured intenaitics changing by a factor of 1 within a few days (Fichtel et al. 1993).

(One of the sources deterked by F(BRET, Mrk 121, has been detected nbove solo) GeV by the Whipple atmonpheric (ierenkov telescope (Punch et al 1002). Asumming that a simple power law energy spertrum connecto these two observations, the intexin! gamma-ray flux from Mrk 421 can be represented na

$$
\phi(\times E) \quad 7.14 \times 10^{12} E^{1.117} \cdot m \text { d }
$$

with $F$ measured in 'TeV.

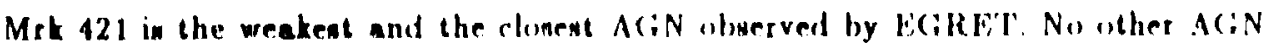
has been obeetved tos emit TeV kamman radintion. Sienttering of 'IeV photuns by an

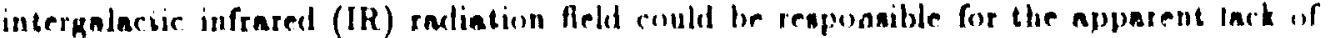

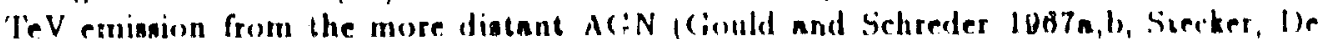
Jager, and Salamon 1902). Siner the intensity nod spectrum of the intergalnctic Ill field ne unknown, in the following nnalyain it is nasumed that there is no nimorption due to no intergalactic Ill raliation firld. 
The properties of the $2.7 \mathrm{~K}$ cosmic microwave background radiation field (C.MBR) are well known. It has been pointed out by several authors (Nikishov 1961, Jelley 1966, Gould and Schreder 1966, 1967a,b) that ultra-high energy (UHE) ( $100 \mathrm{TeV})$ gamma rays will interact with this field and create electron-positron pairs, leading to a cutcff in the spectrum observed a: earth. The calculation of fux upper limits presented here accounts for this effect.

2. TEE CYGNUS EXPERIMENT The CYGNUS air shower array located in Los Alamos, NM, has been described elsewhere (Alexandreas et al. 1991, and Alerandreas et al. 1993). This paper describes the analysis of 350 million events taken with the CYGNUS-I array from 1986 April to 1993 January.

For a source parsing through senith $\left(\delta=36^{\circ}\right)$ the median primary energy for gammaray-initiated eveats is $\sim 70 \mathrm{TeV}$, assuming that cosmic gamma rays and cosmic rays have similar energy spectra. The median detected primary energy increases with senith angle.

The CYGNUS-I array bas two muon detectors. The first muon detector (Allen et al.1992) is a $\sim 44 \mathrm{~m}^{2}$ array of multiwire proportional chambers. The minimum energy required for a muon to penetrate the detector's shielding is $2 \mathrm{GeV}$. The second muon detector was originally a $70 \mathrm{~m}^{2}$ liquid scintillator detector. This detector was used for muon identification in 1987 between January 22 and May 14, and again in 1990 from May to November. In November of 1990 the liquid sciatillator was replaced by 26 scintillation counters (total area $\sim 16 \mathrm{~m}^{2}$ ). The minimum energy required for a muon to penetrate the shielding was $5 \mathrm{GeV}$. In 1991 October the scintillation counters were moved to a shielded room, a minimum muon energy required to penetrate the shielding is $0.7 \mathrm{GeV}$.

3. SFARCE STRATEGY AND RESULTS The search method has been described elsewhere (Alexandreas et al. 1993). 'The source bin spans $2.0^{\circ}$ in declination $(\delta)$ and $2.0^{\circ} / \cos \delta$ in right acenaion. We have searched for emission "rom all of the AGN delected by EGRET that are visible irom our latitude (uee Table 1). Given the presence of both steady and variable components to the emission at lower energies, we have looked for emusion on three different time scales: steady emisuion, emission on the time scale of one week, and eminsion on the time scale of one day. We have also searched for continuous emiscion in the muon-poor (events with sero observed muona) data sampie.

For each day, the obeerved number of events and the expecterd number of background erents in each cource bin are found. This is rxpressed as the number of atandard devia. tions (positive or negative) calculated accurding to the Li and Ma preacription (Li and Ma 1983). To search for emisoion un the time seale of one week, we have summed the daily results for eeven consecutive calendar days, with a new (not atatintically inciepen. dent) weven day period beginning on each day.

'Table 1 gives the observed number of events and the number of experted bachground events for each AGN for the entire deta set and for all showers with wero observed inuons. Table 2 gives typical values for these quantities for the weetly and daily time ncales. No statistically ugnificant excen is observed from any of the $A$ (i) for any of the time scales examined.

The oberrver number of on-soutce eventy and the expected number of bachkround ereate are used to derive a $90 \%(:, I$. upper limit on the nu.aber of excesn events in the noure bin using the preacription of IIelene (Helene 1983;. 'These upper limits, expreamed a * fraction of the conmic-ray bachground in the source bin, are given in the tables $(f(n))$.

'The nil-particle conmic-ray $\|_{u x}$ nbove nn energy $H ;, \phi_{r} \cdot h(\cdot k j)$, in uned lo ronvert

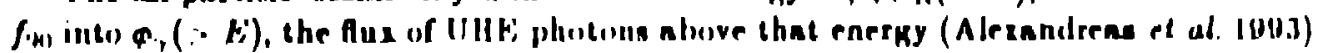

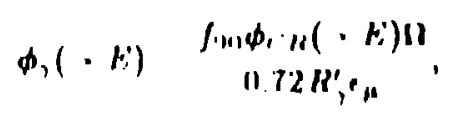

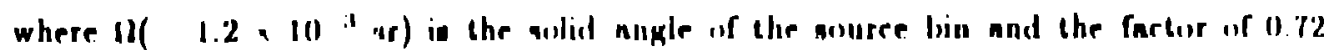
acrounte for the fraction of the signal that is experted to be contained in the suiner 
bin. The factc: $\epsilon_{\mu}$ is the fraction of gamma ray showers which survive the muon cut For the data se: without muon cuts $\epsilon_{\mu}=1.0$. For the muon poor data sample $\epsilon_{\mu}=0.9$ The cosmic ray flux, $\phi_{C R}$, is obtained from Burnett et a!.1990 in the same manner as in Alexandreas et al. 1993.

The factor $R_{\gamma}^{\prime}$ in equation 2 accounts for the different detection efficiencies for gamma-ray and cosmic-ray primaries of the same energy and for the different energy spectra of gamma rays and cosmic rays. To determine $R_{\gamma}^{\prime}$ an $E^{-2}$ differential gamma ray source spectrurn is propagated through the microwave background field (Nikishov 1961, Jelley 1966, Gould and Schreder 1966, 1967a,b, and Stecker, De Jager, and Salamon 1992). For a source at a given redshift this yields a spectrum at the top of the atmosphere. For a close source such as Mrk $421(z=0.03)$ this leads to a cutoff in the spectrum at $\sim 100 \mathrm{TeV}$. For a source with a redshift of 2.2 the spectrum is cutoff at $\sim 10 \mathrm{TeV}$. Equal numbers of gamma-ray showers and cosmic-ray showers are simulated, then $R_{\gamma}^{\prime}$ is the ratio of the number of gamma-ray showers that yield a trigger to the number of cosmic-ray showers that yield a trigger. Since the energy threshold of the detector increases with zenith angle $R_{\gamma}^{\prime}$ is a function of source declination. In addition, the spectrum observed at earth depends on the redshift of the source, so $R_{\gamma}^{\prime}$ depends on the redshift of the source. If the energy threshold of the array is greater than the energy of the spectral cutoff, $R_{\gamma}^{\prime}=0$. Thus the array has almost no sensitivity to gamma rays from sources at large redshifts and large zenith angles.

Table 1 also gives the $90 \%$ C. L. upper limit on the observed flux above the median gamma-ray energy in the source bin, $E_{m}$, for each of the examined sources. Due to the strong dependence of $R_{\gamma}^{\prime}$ on redshift we have not computed flux limits for sources whose redshift is not known. Note that because of the absorption by the CMBR ihese flux limits can not be simply scaled to different energies. Instead our flux limits scale iike the source spectrum at the top of the atmosphere (e.g. the dotted line in Figure 1).

Figure 1 show the messured flux from Mrk 421 together with the upper limits on the flux from these data. Two curves are drawn on the figure. The dashed curve is a direct extrapolation of the lower energy mensurements of the source flix. The dotted curve is the same source spectrum after attenuation of the UHE gamma rays by the $2.7 \mathrm{~K}$ CMBR. The upper limit to the flux at 50 ' $\mathrm{TeV}$ falls between the two curves, indicating that it is consintent with the source spectrum as measured at lower energies with attenuation by the $2.7 \mathrm{~K}$ CMBR. Since the upper limit is above the lower curve, these results are insenaitive to the intergalactic IR field

4. SUMMARY. A search for UHE emission from Mrk 421 and 12 other AciN de tected by EGRET has been described. The search wan performed using data from the CYGNUS experiment from 1986 A pril 2 through 198.3 Jnnuary 1 . No signifirant excess of events in observed from any of these objects for any of the time scales examined. The upper limit on the flux of UHE gamma rny, from Markarian 421 is consintent with the source spectrum meanured by Whipple and BCORET with aborotion by the $2.7 \mathrm{~K}$ CMBR.

ACKNOWLEDGMENTS Several oi us nre grateful to the MP Division of lon

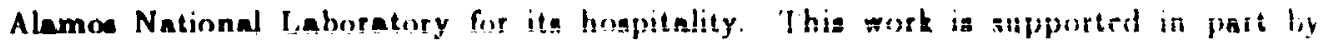
the National Science Foundation, lon Alamon National Labormlory, the U.S. Mrpart ment of Energy, and the Inutitute of (irophyeses arid P'lanetary l'hysics of the Iniversitv of California.

\section{Rofurences}

Alexandreas, D. W., et al. 1891, Nurl. Luetr, and Meth.. As11, 351 .

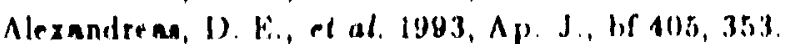

Allen, H. (, , et al.1982. Nucl. Inntr. nud Meth, A $311,30 \mathrm{H}$.

Burnett, 'T. II., +t al lovo, Ap. J. l,ett.,340, .:25

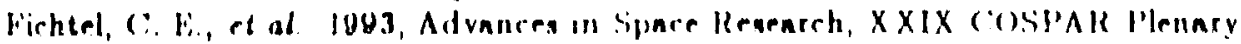

Mretink, in preperation, (New Yort Piorkemon l'rens).

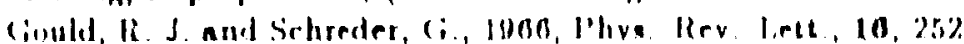


Gould, R. J. and Sehreder, G., 1967a, Phys. Rev.,155, 1404.

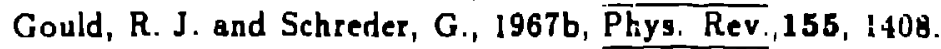

Hartman, R. C. et al, 1992, Ap. J. Lett., 385, Ll.

Helene, $O ., 1983$, Nucl. Instr. and Meth.,212, 319.

Jelley, J. V., 1966, Phys. Rev. Lett., 16, 47 .

Li, T. P. and Ma, Y. Q., 1983, Ap. J., 272, 317.

Vikishov, A. I., 1961, 2h. Eksp. i Teor. Fiz., 41, 519 English Trans. Sov. Phys. JETP, 14, $393(1962)]$

Punch, M., et al. 1992, Nature, 368, 477.

Stanev, T., Gaisser, T.K, and Halaen, F. 1985, Phys. Rev. D, 32, 1244.

Sitecter, F. W. De Jager, O. C., and Salamon, .H. H., 1992, Ap. J. Lett., 380, L49.

\begin{tabular}{|c|c|c|c|c|c|c|c|c|c|c|}
\hline Bource & 2 & $.6 m$ & $V_{011}$ & S:. & D. & $V_{i n}$ & $N_{1}^{2}$ & $\rho_{-1.1}^{p}$ & $6 !$ & $T . n$ \\
\hline $11202+1191(1)$ & & .56022 & $\overline{5426}$ & 111111 & & 26.134 & 26173 & $(1.140$ & $\cdots$ & \\
\hline ['KSiO2:15 (2) & 0.94 & $6(1646$ & 60:3): & 11.111 & 316 & $2+4066$ & $28.59-1$ & $(1,111)$ & 20 & $\vdots \prime \prime$ \\
\hline PKSOL'20 (3) & 0.92 & $13.3 . \% 0$ & 1.1205 & 11,117 & iv. 1 & 312.1 & 1911 & 11.015 & 26.7 & 211 \\
\hline PKSOS28 (-1) & 2.07 & 52015 & 522119 & 1) III & . & 2111.5 .5 & 21915 & $(1.1)(16$ & $\ldots$ & \\
\hline $11716+71+(5)$ & $\cdots$ & 28596 & $2 \times \cdot 11)^{2}$ & $11.111 ;$ & . & 1211.5 & 12022 & 01.1110 & $\cdots$ & \\
\hline $1 C+71.107(6)$ & 2.17 & 29255 & $2(1251)$ & $11,11)$ & . & $12: 410$ & 12:376 & 11.005 & $\ldots$ & $\cdots$ \\
\hline Mrk $\cdot 12 !(7)$ & 0.0 .31 & 1034332 & IU:17! & 111116 & 1119 & 57198 & 572114 & $0.0(0) .1$ & 19.173 & il) \\
\hline$(10: 273, H)$ & 1). 158 & 9816 & 91272 & 1110.5 & 11.13 & iAnI & Hu75 & $(1.005$ & 0.099 & i1) \\
\hline IC279 (4) & $1) .5 .28$ & 7152 & 7:1:16 & 111111 & ز & $2|8|$ & 25.35 & II.(N)H & A.I & 2.5 \\
\hline PKSIG(16 (10) & 1.23 & 11699 & IIthit & HINA & $\therefore H$ & IHGHS & IHAIO) & $0.0 \times 4$ & h. 2 & $1 ;$ \\
\hline $1 C+.18 .+1(11)$ & 1.81 & 101839 & $1(1) \mathrm{H}() .1$ & "III) & 11.6 & 35863 & .56 .195 & $0 .(x) 2$ & 5. 1 & 111 \\
\hline $10+11.69(12)$ & 1.037 & $\$ 4956$ & $\mid-1 \cdot 11)\}$ & "1111 & 20.7 & $20: 362$ & 20117 & 0.010 & 12.1 & 15 \\
\hline $30.15 .1 .3(1.3)$ & 1).859 & 59606 & itilfis & $11111)$ & -2.17 & $2454 \pi$ & 27988 & 0.010 & $2 . .1$ & 20 \\
\hline
\end{tabular}

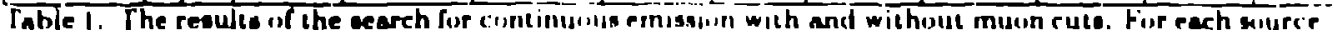

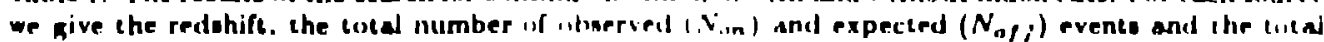

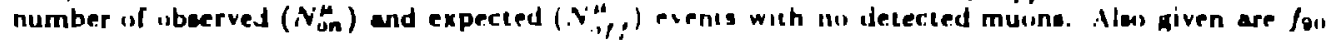

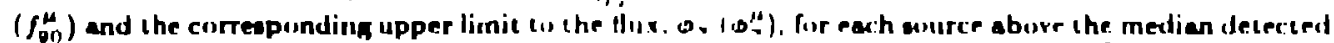

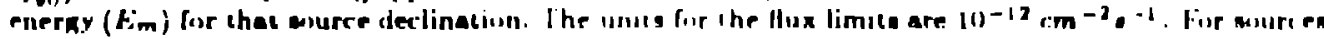
whowe redohift is eicher unavelable ur tou large wr i no nuc compute a thu limit.

\begin{tabular}{|c|c|c|c|c|c|c|}
\hline \multirow[b]{2}{*}{ 5* } & \multicolumn{3}{|c|}{ Tally Resules } & \multicolumn{3}{|c|}{ THerbly Tranilie } \\
\hline & $\operatorname{Vim}$ & $\mathbf{v i y l}$ & J00 & $\nabla_{10 n}$ & 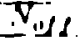 & $T_{311}$ \\
\hline 1 & 11 & 11.0 & $0 . \overline{x 0}$ & 280 & $278 \pi$ & $1 i i$ \\
\hline 2 & 17 & 12.3 & 0.38 & 2406 & 29:4.H & | I \\
\hline .1 & H & \$. $\mathrm{B}$ & 11.53 & 6.1 & (i2.1) & II IH \\
\hline 1 & Is & 33.2 & 0.32 & 271 & 254.1 & 1111 \\
\hline $\mathbf{s}$ & 211 & 23.1 & (0.:21 & 141 & $(1,3 !), 1)$ & 11.1 .1 \\
\hline 6 & $2 \theta$ & 23.3 & $0.5 n$ & IRI & 130.19 & 11.1 .1 \\
\hline 7 & 94 & (x). & 11.22 & 51.5 & 5.17 .1 & 11.11 .5 \\
\hline M & I I & 14.4 & 0.139 & m & 18 & 1117 \\
\hline$!$ & A & 3.8 & 1.10 & in & .11 .1 & ו" \\
\hline 118 & :II & H.4 & 0.27 & IA2 & 212.7 & $11: 17$ \\
\hline 11 & 79 & 70.1 & 11.21 & 494 & 5272 & $11116 ;$ \\
\hline 12 & 15 & .21.8 & 0.10 & 247 & 215.1 & 11.3 \\
\hline 13 & so & 42.5 & 0.44 & $2 \mathrm{~m}$ & 24.95 & "M \\
\hline
\end{tabular}

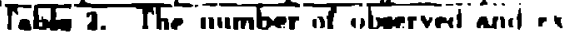
peceed evente lirr each wurre examined lin' a typied day a...l a typical week. Nlaw miven

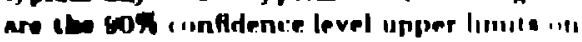
the frection of exires evente in the sullite

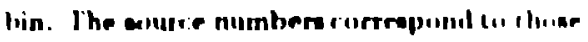
given in Iable 1.

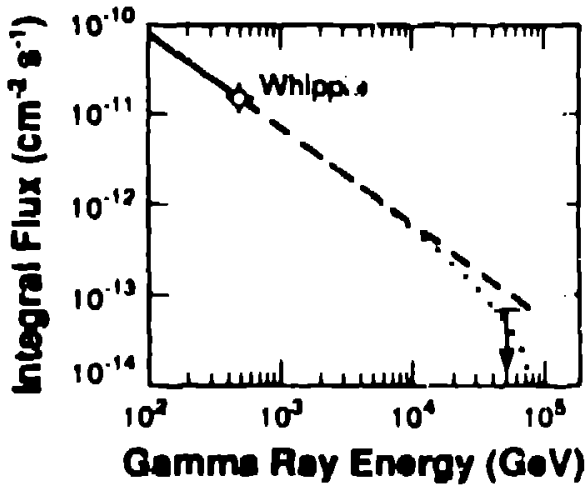

Figure 1: 'The iategrel ramme-tay fux from Marbarinn 1'! meapured by KiGRE'T and the Whipple leleacope lor gether with the muon poor upper lumit frnnt the CYGNUS experiment. The dolled (dnahid) curve corresponds to the expected opuitum at earth with (no) abeorption by the CMBR. 